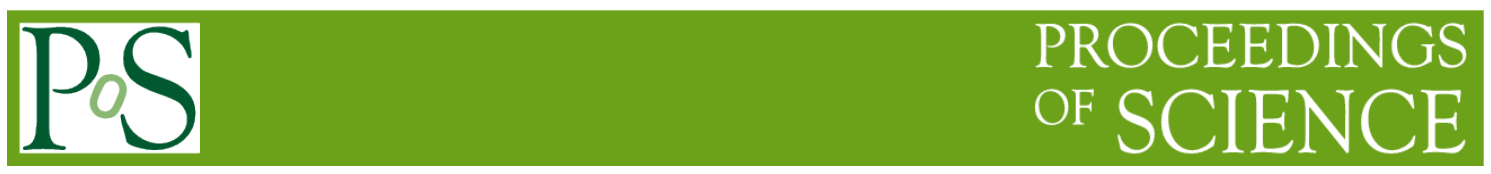

\title{
An Unified Payment Method of Charging Piles Based on Blockchain
}

\author{
Junsheng Wang ${ }^{1 a}$, Qingsu $\mathrm{He}^{\mathrm{b}}$, Yu Xuc ${ }^{\mathrm{c}}$, Qingzhi Han ${ }^{\mathrm{d}}$ \\ Beijing Huitong Financial Information Technology CO. LTD \\ Beijing 100032, China \\ State Grid Electronic Commerce Co. LTD \\ Beijing 100032, China \\ E-mail. ${ }^{a}$ wangjunshengesgitg.sgcc.com.cn; ${ }^{b}$ heqingsuesgitg.sgcc.com.cn; \\ c2443835553@qq.com; ${ }^{d}$ hqz1245086050@126.com
}

\section{Ziqiang Zhou}

Zhejiang Electric Power Corporation

Hangzhou 310007, Zhejiang

E-mail: jx_zzq@sina.com

In order to reduce the dependence on oil,develop clean energy, alleviate environmental pollution and other issues, the electric car, as the representative of the new energy industry, has been greatly supported by the government and achieved vigorous development. Blockchain is an innovative revolution over the internet finance; however, the current trading mode involves the third party. As a result, the cost has increased while accompanied with reduced efficiency. In this article, in respect of the problem between different current charging piles not to achieve unified charge payment, we use the consortium blockchain to establish an unified payment channel without involvement of the third party. It can solve the problems such as great inconvenience to users caused by different charges and payment methods in different charging pile operators, and thus promote the rapid popularization of electric vehicles.

CENet 2017

22-23 July 2017

Shanghai, China

${ }^{1}$ This research is supported by the Science and Technology Project of State Power Grid Corp (No. 52110417000G). 


\section{Introduction}

With the gradual improvement of people's living standards recently, the car has become the travel vehicle for more people; at meanwhile, the enormous cars have brought about a series of problems such as energy consumption and environmental pollution, etc.. In face of the dual pressures of environmental pollution and energy crisis, countries around the world compete to develop alternative fuel vehicles. With the advantages of zero emission, energy diversity and easiness of control, electric vehicles have become the object that governments encourage such development. In order to generalize the application of electric vehicles, China has promulgated a variety of policies to support the development of electric vehicles and invested more in charging facilities construction since 2014. The National Energy Administration said on February 9, 2017," "China will strive to add 800 thousand charging piles including 700,000 special piles and 100,000 public piles". In 2017, the Ministry of Industry and Information Technology and National Energy Administration will further strengthen the infrastructure construction of charging piles and encourage building the piles by means of crowdfunding.

However, with the rapid development of the charging pile industry, for the actual operation of the charging pile, various operators have established their own payment platform, developed different charging standards and diversified payment methods, which have not only resulted in great inconvenience for users in terms of the charge payment, seriously reduced the desire of consumers to purchase electric automobiles, and stunted the growth of electric cars industry. What is more importantly, it has declined the utilization of the charging pile. With the development of the blockchain, it can be applied to the field of electric vehicle charging to build unified payment channels so that the users may charge and pay easily.

With regard to the significant advantages, such as transparency, reliability, decentralization and other characteristics of the blockchain, it can ensure the mutual trust between different companies and thus greatly reduce the cost of maintaining trust. In view of the superiority of technology, many scholars have carried out research and exploration actively [1-4]. The design demand has explored and analyzed deeply [5], the consistency and the Scalable applications of blockchain technology. Based on the research on the application system development method and modeling of the blockchain technology, the account blockchain and the trading blockchain were proposed. One Paper, based on the applications to the economy of the blockchain and the financial payment as an example, elaborated the traditional payment model based on difference and block chain technology, payment mode [6]. And it proposed the essence of the application of blockchain technology in the financial field to build a flattened global integrated liquidation system. On the basis of analyzing the necessity of the application of blockchain technology in China's paper market and the development constraints, the paper puts forward the overall development ideas and suggestions in respect of the application of block chain technology in China's market [7]. One Paper, based on the blockchain technology, studied the distributed personal data management system to ensure the user management and control of their personal data [8]. In addition, the research of the blockchain technology has been extended to the Internet of Things [9], artificial intelligence[10] and other fields. The German RWE group has verified the electric car' payment system based on blockchain technology, but the specific technical details are not publicly disclosed.

Blockchain is divided into three different groups: the public blockchains, the consortium blockchains and the private blockchains. The consortium blockchains can make perfect connection between a node and another nodes, and thus enable peer-to-peer transaction, 
coordination and collaboration in the system so that there's no need for all nodes to trust each other. In this sense, it is effective to improve the efficiency of transaction processing and reduce the cost of transaction. What's more, it is able to solve the problem of block expansion and has strong expandability. In this paper, the consortium blockchains is used to study the problem of uniform payment for charging pile so that the current users can easily recharge and pay. At meanwhile, the utilization of charging pile can be improved.

\section{Development Status of the Charging Pile}

In 2016, China gave great impetus to the construction of rechargeable piles. the number of charging piles were added from almost 50000 at the beginning to 150,000 at the end of the year with an increase of six times compared to that last year. The special charging piles have been increased by more than $80 \%$ over the same period last year. At present, there have been more than 300 companies selling charging piles, including more than 40 listed companies about charging facilities. In addition, there are multiple market segments in the industry chain, including the charging equipment spare parts supply, the charging facilities operations, the integrated facilities services, etc.. As these companies are decentralized relatively, it is not enough to gather into the scale economy. But in recent years, with the rapid development of the charging pile industry, the complete industrial chain including upstream equipment manufacturers, midstream charging operator, downstream total solution provider has been gradually formed [11]. The State Grid will make the overall layout of the charging network operation by establishing the "seven vertical and four horizontal two grid" highway fast charging network on the basis of highway intercity fast charge network of the "two vertical and two horizontal and one ring" including the highway of Beijing-Shanghai, Beijing-Hong Kong and Macao, Qingdao-Yinchuan, Shanghai-Chengdu, Nanjing-Shanghai and Hangzhou. Aiming at different degrees of the popularity of the clean-energy vehicles, China is to construct the charging facilities by accelerating and actively promoting the development and demonstration and popularization of the electric vehicle. As supported by the government policy and incented by the multiple benefit, the market of charging piles will accelerate its progress.

However, "China's electric car industry is still on the initial stage of development. As the proverb goes "Sail against the current, fall behind", although the scale of its production and sales market is growing rapidly, the growth of construction of charging facilities is relatively slower, and demands acceleration", Mr. Miao Wei said. At meanwhile, the charging piles are not compatible with the electric car purchased by the user, which leads to poor user experience. The difficulty in creating profits for the enterprises involved in charging pile forms the radical cause that may hinder the development of electric vehicle industry. Even some charging pile operators indicated that they've invested great money and time, but achieved nothing.

At present, the main problems of charging pile industry in China are shown as follows: (1) as the construction of charging facilities is slow, to some extent, which has become an important factor restricting the development of electric vehicle industry; (2) among the charging piles that had been built, various operators have established their own payment platform, developed different charging standards and payment methods, which results in non-interoperability between each charging pile; (3) the technology research and development capabilities need to be improved, such as the high-power charging technology, the wireless charging technology and the high-performance power battery technology; (4) the high pre-construction cost and coordination difficulty between companies impede the development of the operation mode of 
charging pile business, and there is no distinct profitable measures; (5) the industrial standards are imperfect in lack of criteria of unified and perfect acceptance and supervision.

\section{The Model of Consortium Blockchains about Charging Piles}

Suspendisse: the consortium blockchains with polycentric structure, including the transaction node and billing node. Each charging pile as a trade node can achieve the charge payment and the interaction function of transaction information and network; each company has a billing node as a centre of the polycentric structure responsible for providing accounting services to the entire network and maintaining global books.

The billing node is the core of the consortium blockchains according to the functional definition. All enterprises in the alliance need to pay a certain margin to form the initial pool of funds, which can be thus used to reward the billing node generating a new block and the promotion of the league.

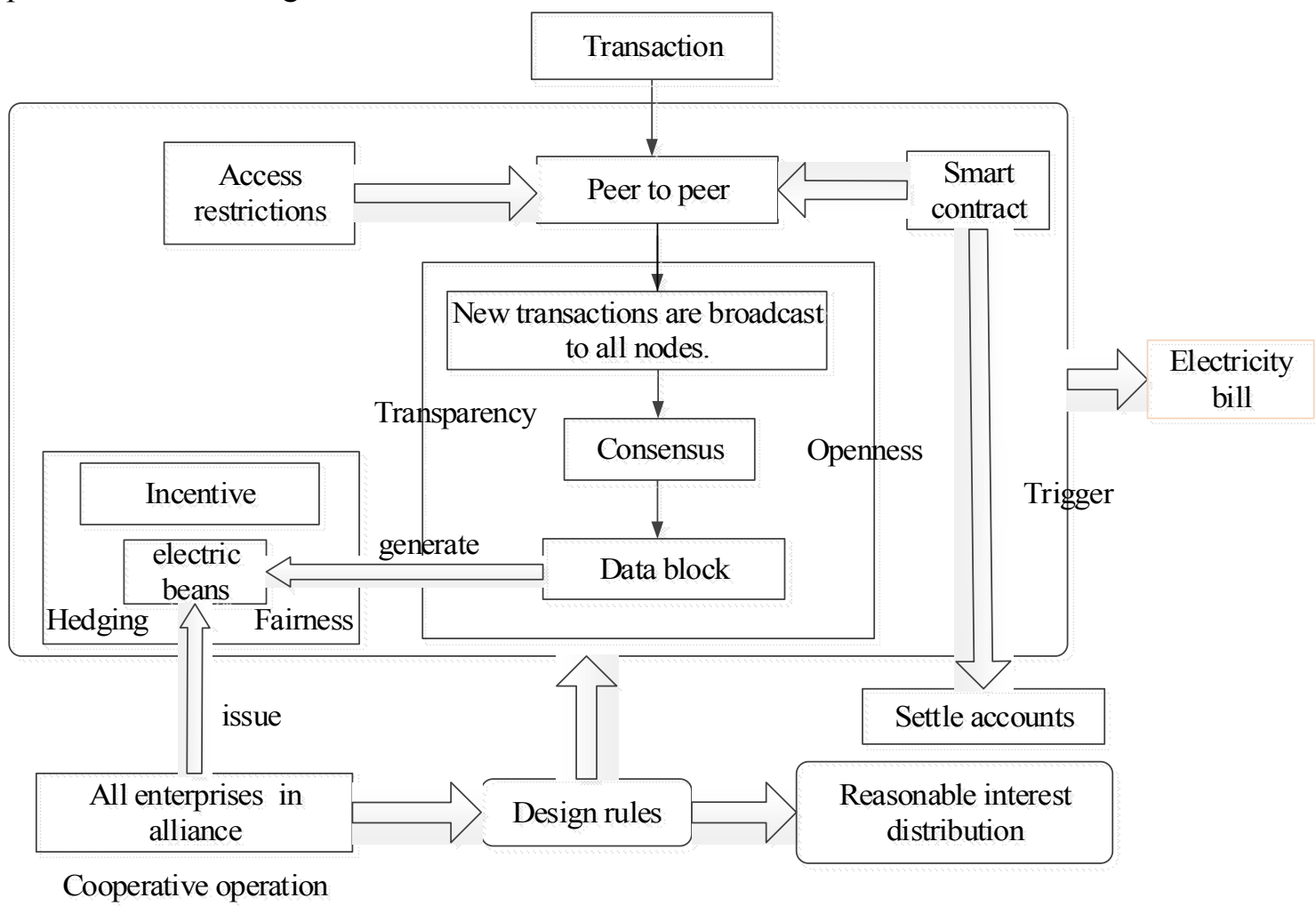

Figure 1: The Hierarchy Model of Charging Pile Union Chain

Consortium blockchains consists of the data layer, the network layer, the consensus layer, the incentive contract layer, the layer and the application layer, in which the data layer encapsulates the underlying data blocks and the technologies of related data encryption and timestamps and other technologies; the network layer mainly includes the distributed netting mechanism, the data transmission mechanism and the data validation mechanism; the consensus algorithm of the network node is encapsulated in the consensus layer; the economic factors are integrated into the blockchain system by the incentive layer; the contract layer encapsulating all kinds of scripts, algorithms and intelligent contracts forms the basis of programmable characteristics of the blockchain; and the application layer encapsulates the various application scenarios of the unified payment of power charge of the charging pile. The hierarchical model is shown in Fig. 1. 
The data layer and the network layer of the charging pile consortium blockchains adopt the linked storage structure and $\mathrm{P} 2 \mathrm{P}$ network protocol similar to that of the bitcoin; however, the encryption algorithm has been modified by the national algorithm not based on SHA256 [12] but the national algorithm SM3; the consensus layer utilizes the Byzantine fault tolerant consensus algorithm; the contract layer is programmed based on the standardized contract template [13].

\section{Transaction Process and the Electrovalence Competition Strategy}

At present, Proof of Work (PoW), Proof of Stake (PoS), Delegated Proof-of-Stake (DPoS), Ripple Consensus and Practical Byzantine Fault Tolerance are alternative. As each billing node of the consortium blockchains can record accurate and reliable transaction data, the consensus algorithm mainly solves the fault tolerance problem in this paper.

The Byzantine fault tolerant algorithm (BFT) can tolerate any form of software bugs and security vulnerabilities. It is a general algorithm to solve the problem of fault-tolerant distributed system. For example, in a distributed system, with $M$ nodes, the algorithm can guarantee that when all nodes participate in the process of the consensus, then some nodes make mistakes but less than or equal to (M-1)/3, the system still maintain security and activity. The detailed derivation and step for the BFT algorithm are written [14-16] .

The consensus mechanism based on Byzantine fault tolerance has been applied to the blockchain, for example, IBM introduced PBFT [17] at Hyperledger fabric, Onchain releasing antshares consensus algorithm dBFT (delegated BFT) [16]. Different copies among linked data structure of the traditional blockchain cannot always keep consistent, which makes the block to reach different nodes at different times, resulting in blockchain bifurcation phenomenon. However, if we adopt the BFT consensus mechanism, it will not be bifurcated within the fault tolerance [16].

Although the processing capacity of BFT is far less than that of PoW and some other consensus mechanism, the capacity of the data transaction and processing communication overhead of the nodes participating in the consensus process is very considerable with its overhead being polynomial growth [15]. The charging pile consortium blockchains need to ensure the generation velocity of the block to save the payment time for a strong user experience. All billing nodes in the consortium blockchains should participate in the consensus process and generate the corresponding blocks to guarantee the accuracy and trustworthiness of the trading data. The process is as follows.

(1) New transactions from transaction nodes are broadcasted to all billing nodes.

(2) Each billing node collects new transactions into a block.

(3) All of the billing nodes should record the transactions as per a certain sequence such as sorted by the first letter of the company's English name. After the last record has been completed, the next node which records the new transactions information is the first billing node, and so on.

(4) Every $t$ second (for example, $t=1$ ), the current billing node broadcasts the block to all billing nodes. The billing nodes accept the block only when all the transactions therein are valid, and the billing nodes express their acceptance of the block by creating the next block for it; otherwise, no new block is generated. If the new block is generated, a transaction free from the initial pool of funds is added as the reward.

(5) Billing nodes express their acceptance by Byzantine fault tolerance algorithm. 
The flow diagram is shown in Fig. 2.

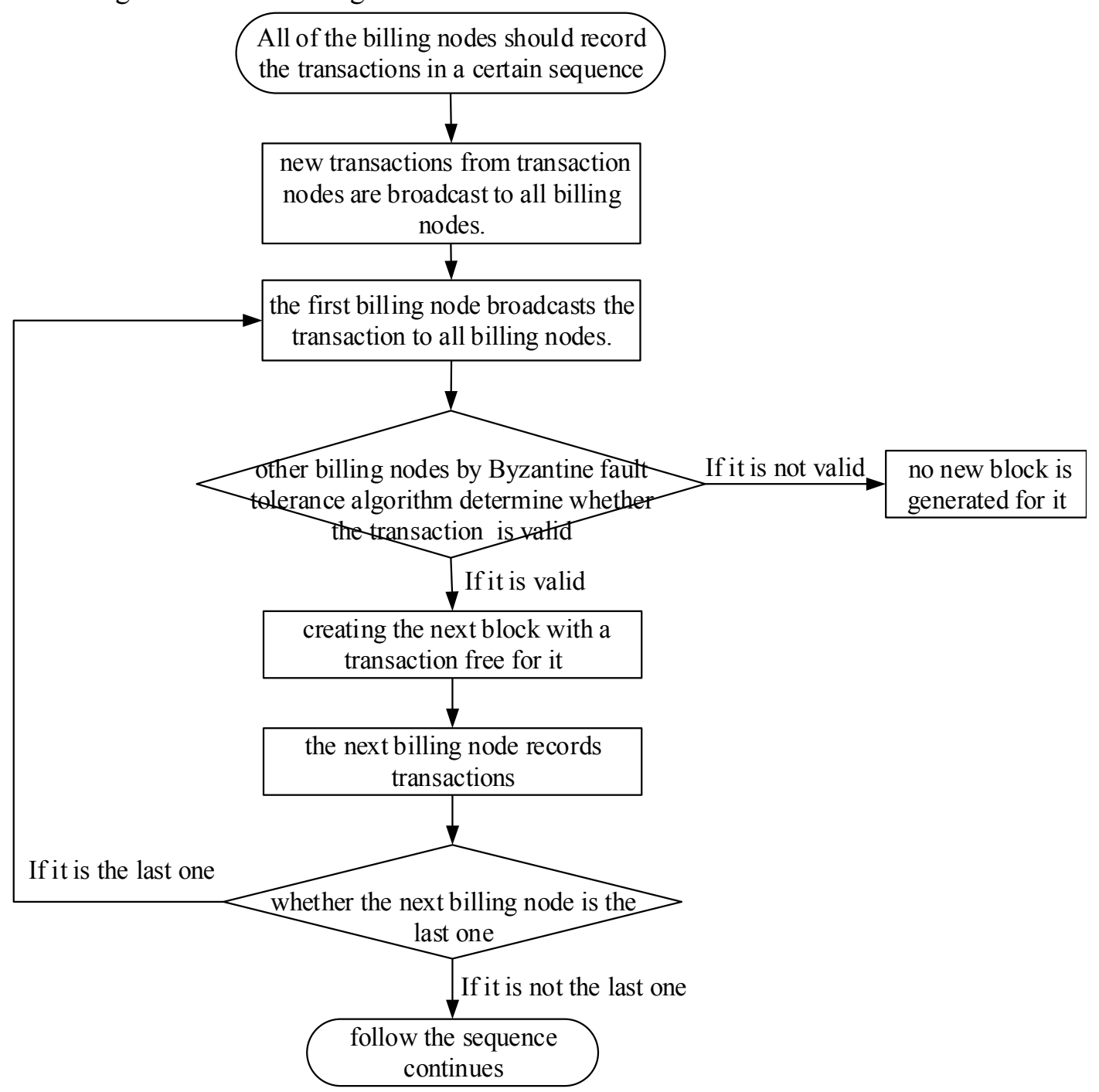

Figure 2: The Flow Diagram of Keeping Accounts

Because the relationship between the enterprises of the consortium blockchains are both cooperative and competitive, each of them will strive to allow more users to charge their own charging pile. In addition, due to different operating costs and business strategies, fee standards are also diverse, which will make the checkout process complex and "price confusion" for users.

In order to solve the above problems, the incentive strategy about electrovalence competition is adopted in this paper.

(1) The charging piles that different enterprises in the alliance manage can be distinguished by various identifiers;

(2) The electrovalence of different enterprises should be the same;

(3) Each enterprise, according to their own needs, issues the corresponding number of "electric beans" on the basis of the achieved reward;

(4) The "electric beans" are distributed to the users by means of charging rebate, selling or dispatching red envelopes. The "electric beans" can be equivalent to the electrovalence, for example, one "electric bean" is equivalent to once electricity but only limit to the charging piles of the companies who give "electric beans" to the users. 


\section{Conclusion}

With the continuous development and the deepening applications of blockchains, blockchains 2.0 represented by the intelligent contract and decentralization application (DAPP) is gradually applied to financial services, supply chain management, culture and entertainment and other fields. As an important type of application of blockchain technology, the consortium blockchains has been widely concerned for its scalability and low risk. In this paper, based on the payment scenario of electric car charging, the blockchain is adopted to solve the problem of users' cumbersome charging payment or recharging in hopes that it will be helpful for the research and application of relevant direction.

\section{References}

[1] M. Swan. Blockchain thinking: the brain as a decentral-ized autonomous corporation[C]. IEEE Technology and Society Magazine, 2015, 34(4): 41-52

[2] E. Davidson. Letter[J]. New Scientist, 2015, 228(3043):52-52

[3] Anonymous. New kid on the blockchain[J]. New Scientist. 2015, 225(3009):7

[4] P. Godsiff. Bitcoin: bubble or blockchain[C]. In: Proceedings of the 9th KES International Conference on Agent and Multi-Agent Systems: Technologies and Applications (KES-AMSTA). Sorrento, Italy: Springer, 2015, 38: 191-203

[5] W.D.Cai, L.Yu, R,Wang, N,Liu, E.Y.Deng. Blockchain Application Development Techniques[J]. Journal of Software, 2016, Network priority publishing address: http://www.cnki.net/kcms/detail/11.2560.TP.20170222.1047.004.html

[6] S.Wang. Research Status and Innovation Trend of Blockchain Technology in Financial Field [J].Shanghai Finance, 2016, (2):26-29

[7] A.J. Ren. Thoughts on The Reform of China's Bill Market by Using Blockchain[J]. South China Finance, 2016, (475):39-42

[8] G Zyskind, O Nathan, A.S.Pentland. Decentralizing privacy: using blockchain to protect personal data $[\mathrm{J}]$. In: Proceedings of the 2015 IEEE Security and Privacy Workshops (SPW2015). San Jose, CA: IEEE, 2015.180-184

[9] W.Ding. Block Chain Based Instrument Data Management System[J]. China Instrumentation, 2015, (10): 15-17

[10] H.Zhao, X.F.Li, L.K.Zhan, Z.C.Wu. Data integrity protection method for microorganism sampling robots based on blockchain technology[J]. Journal of Huazhong University of Science and Technology (Nature Science Edition), 2015, 43:216-219

[11] Y. H. W, J. M. Research on Present Situation and Problem Countermeasure of Charging Pile[J]. China Market Marketing, 2016(41):45-45

[12] S. Nakamoto. Bitcoin: A Peer-to-Peer Electronic Cash System[Online],available: https://bitcoin.org/bitcoin.pdf

[13] The white paper: China blockchain technology and application development (2016)

[14] M.Castro, B.Liskov. Practical Byzantine fault tolerance and proactive recovery[J]. ACM Trans.on Computer Systems, 2002, 20(4):398-461

[15] J.Fan, L.T.Yi, J.W Shu. Research on the Technologies of Byzantine System[J]. Journal of Software, 2013(6):1346-1360 
[16] Z.W.Zhang. A Byzantine Fault-Tolerant Algorithm for Block Chains(2016)[Online], available: http://www.onchain.com/paper/66c6773b.pdf.

[17] Hyperledger Whitepaper [Online], available: Wechat ID: Blockchain Pencil (2016). 